\section{口 BRÈVES}

nue. Devant l'impossibilité de mettre en évidence un ADN responsable, on a surtout discuté du pouvoir infectieux de protéines appelées prions (voir $\mathrm{m} / \mathrm{s} n^{\circ} 2$, vol. 7, p. 186). Un chercheur de Newcastle (RoyaumeUni) ouvre une voie intéressante par des méthodes morphologiques. Le travail de Narang [1] est fondé sur l'examen de 50 hamsters inoculés avec une suspension de cerveaux infectés qui provoque une tremblante clinique en deux à trois mois. Le cerveau de ces animaux présente des particules de tubulofilaments. Si on prépare l'ADN et qu'on l'examine en microscopie électronique après étalement sur des grilles recouvertes de collodion, on voit surtout, chez les malades comme chez les témoins, des molécules d'ADN double brin d'environ $16 \mathrm{~kb}$ qui ont tous les caractères de l'ADN mitochondrial. Mais en plus, chez les malades seuls, on peut voir des molécules d'ADN simple brin, dont on peut évaluer, d'après leur taille, le poids moléculaire à environ $0,5 \times 10^{6}$. Ni les témoins ni des animaux porteurs d'autres encéphalopathies n'ont montré unc telle image. Ces molécules résistent à l' ARNase, mais sont digérées par une combinaison de protéase et d'ADNase. Cette dernière enzyme seule est inefficace. On peut donc admettre que l'ADN monocaténaire mis ici en évidence est protégé par une couche de protéines. Si l'ensemble ADN-protéine est nécessaire à l'infectivité, on conçoit que cellc-ci puisse disparaître sous l'action d'agents qui détruisent ou inactivent les protéines, mais non sous celle des nucléases, qui n'auraient pas accès à l'ADN enfoui dans son manteau protéique. Ce travail est purement morphologique et la nature de l'ADN et de la protéine n'a pas été déterminée. Il peut cependant constituer un point de départ pour une attaque décisive sur la pathogénie de ces redoutables maladies.

[1. Narang HK. J Mol Biol 1990 ; 216: 469-73.]

\title{
Empreinte parentale : expression exclusive du gène maternel du récepteur d'IGF-2
}

Un ensemble d'observations remarquables effectuées ces dernières années [1, 2] a permis de mettre en évidence le fait que, chez la souris et sans doute chez les mammifères en général, le développement embryonnaire ne peut se dérouler normalement que si les cellules de l'embryon contiennent à la fois un génome d'origine maternelle et un génome d'origine paternelle. En d'autres termes, aucun embryon uniparental, parthénote (avec des chromosomes d'origine uniquement maternelle) ou androgénote (avec des chromosomes d'origine uniquement paternelle) ne se développe à terme.

On en a déduit que les génomes paternel et maternel subissent une " empreinte " entraînant une expression différentielle de certains gènes essentiels selon qu'ils sont portés par un chromosome d'origine paternelle ou maternelle. La nature de l'empreinte, le mode de régulation qu'elle entraîne restent cependant à découvrir. Il est donc essentiel d'isoler les gènes soumis à cette empreinte et de démontrer leur rôle dans le développement. S'il a été montré que certains transgènes paraissent se comporter, au moins approximativement, comme on l'attendrait de gènes soumis à l'empreinte parentale [3], aucun gène endogène subissant ce type de régulation n'avait été repéré jusqu'à présent. D'où l'intérêt de l'article de Barlow et al de Vienne (Autriche), Tübingen (Allemagne) et Nashville (TE, USA) paru dans le numéro de Nature du 3 janvier 1991 [4], décrivant la caractérisation d'un gène murin dont l'expression dépend de l'origine parentale du chromosome qui le porte. Les auteurs ont mis à profit l'existence de deux mutations $\mathrm{T}^{\text {hp }}$ et $\mathrm{t}^{\text {Lub2}}$, des délétions chevauchantes situées dans la même région du chromosome 17. Ces mutations entraînent la mort de l'embryon qui les porte, mais seulement si elles ont été transmises par la mère. Le comportement de ces mutations s'interprète aisément si l'on admet que le locus délété contient un gène qui ne s'exprime que s'il est porté par le chromosome d'origine maternelle et donc est soumis à unc cmpreinte génomique parentale. C'est exactement ce qu'orit trouvé les auteurs en combinant de manière élégante une approche génétique et moléculaire, ce qui a posteriori rend encore plus judicieux le nom qui avait été donné à ce locus: Tme pour $T$ associated maternel effect.

Ils ont d'abord observé qu'un gène, Tcp1, est délété dans les deux chromosomes $\mathrm{t}^{\text {Lub-2}}$ et $\mathrm{T}^{\text {hp }}$ et que son équivalent humain est très lié sur la carte génétique à trois autres gènes : ceux du plasminogène $(P l g)$, de la superoxyde dismutase mitochondriale (Sod-2) et du récepteur de l'IGF-2 (insulin-like growth factor 2) (IGF-2-r). La localisation de ces trois gènes a alors été effectuée sur le chromosome de souris et a permis d'établir les bornes de la délétion $\mathrm{t}^{\mathrm{Lub}-2}$ (la plus petite des deux délétions) définissant ainsi une région minimale où se trouve le locus Tme. Le résultat de cette analyse est indiqué sur la figure 1 et montre que les gènes $I G F-2-r, T c p 1$ et $\operatorname{Sod}-2$ sont délétés dans le chromosome $\mathrm{t}^{\text {Lub-2}}$, un gène supplémentaire Plg étant délété dans le chromosome

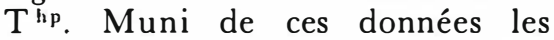
auteurs ont effectué les deux croisements réciproques $\mathrm{T}^{\mathrm{hp}} / \mathrm{x}$ 


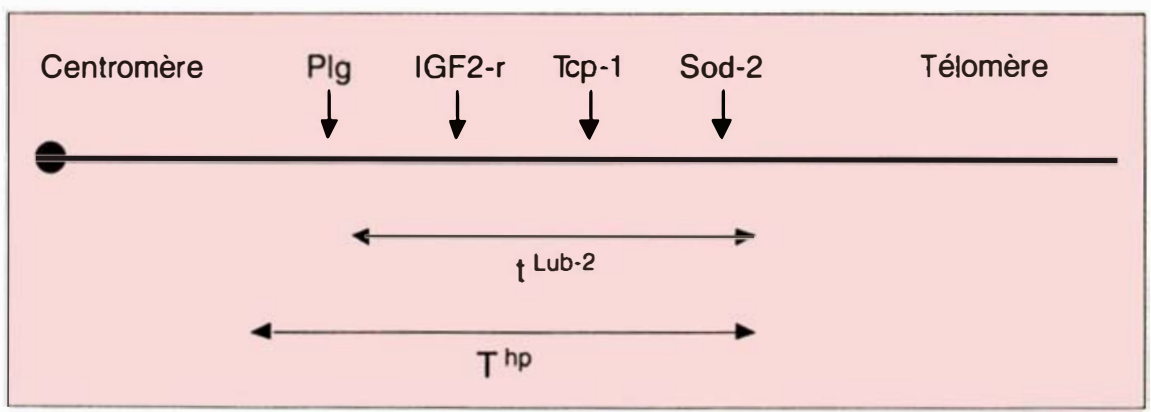

Figure 1. Carte de la région délétée dans les chromosomes 17 de tlub2 et Thp.

$+1+\left(n^{\circ} 1\right)$ et $+/+x$ $\mathrm{T}^{\mathrm{hp} / \mathrm{H}}\left(\mathrm{n}^{\circ} 2\right)$ et analysé l'expression de ces quatre gènes dans les embryons F1. Le résultat est saisissant (Tableau I) : si pour les trois gènes Sod-2, Tcpl et $\mathrm{Plg}$, l'expression observée est indépendante du sens du croisement et reflète le nombre de copies des gènes portés par les embryons $F 1$, en revanche le gène $I G F-2-r$ est totalement silencieux chez les embryons ayant reçu la délétion de leur mère, et ce en dépit de la présence d'un allèle paternel normal. Il faut noter de plus que le niveau d'expression d'IGF-2-r est identique dans les embryons $+/+$ et $+/ \mathrm{T}^{\mathrm{hp}}$ dans le croisement $n^{\circ} 2$. L'ensemble de ces données démontre que le gène de l'IGF-2-r est soumis à l'empreinte parentale ct n'est exprimé que s'il est porté par un chromosome maternel. Ces résulats sont très importants pour deux raisons : d'une part, ils prouvent que le type de régulation génétique postulé pour expliquer le comportement des embryons de souris uniparentaux existe, d'autre part, ils ouvrent la voie à l'élucidation des mécanismes moléculaires de l'apposition de l'empreinte, de sa nature et enfin de son rôle dans l'expression génique.

Des résultats antérieurs, avec une mutation de l'IGF-2 obtenue par recombinaison homologue, suggéraicnt que ce gène est aussi soumis à une empreinte parentale (voire note ajoutée aux épreuves) [5]. En effet les individus hétérozygotes porteurs de cette mutation, et l'ayant reçue cette fois-ci de leur père, sont environ deux fois plus petits que leurs con$\mathrm{m} / \mathrm{s} n^{\circ} 3$, vol. 7 , mars 91 génères et produisent seulement $10 \%$ de l'ARN messager IGF-2 synthétisé par les individus sauvages. Si ce résultat était confirmé par des croisements réciproques, cela signifierait, résultat intrigant, que l'IGF-2, ainsi que son récepteur présumé l'IGF-2-r, subissent l'cmpreinte parentale mais en sens inverse, l'un s'exprimant lorsqu'il est porté par le chromosome paternel (IGF-2), l'autre lorsqu'il cst porté par le chromosome maternel (IGF-2-r). T. Moore et D. Haig, deux chercheurs anglais de Londres et Oxford ont une théorie pour expliquer l'existence d'cmpreinte parentale chez les mammifères et certains végétaux, mais non chez les ovipares, théorie purement spéculative à ce stade mais s'accordant étrangement bien avec les empreintes des gènes de l'IGF-2 et de son récepteur [6]. Selon ces auteurs, les empreintes de gènes paternels et maternels résulteraient d'une sélection pour des caractéristiques antagonistes : faciliter le développement d'importantes annexes embryonnaires assurant le développement de gros embryons du côté paternel, limiter ce développement embryonnaire en limitant les échanges, qui se font d'une certaine manière au détriment de la mère, du côté maternel. IGF-2 pourrait stimuler la croissance embryonnaire, d'où l'existence d'un gène paternel actif.

Le récepteur de l'IGF-2, qui est aussi le récepteur indépendant des cations du radical mannose 6-phosphate des enzymes lysosomiales et qui n'est peut-être pas lié du tout à la transmission d'un signal de croissance, pourrait au contraire intervenir dans l'internalisation et l'inactivation d'IGF-2 dont le rôle physiologique passerait par la fixation au récepteur d'IGF-1.

Selon ce schéma, ce récepteur scrait plutôt un inhibiteur de croissance embryonnaire... dont le gène actif serait uniquement celui d'origine maternelle. Il faut noter, cependant, que les embryons ayant hérité de la mutation Tme de leur mèrc meurent au $15^{\mathrm{e}}$ jour de développement sans grande anomalie de croissance [4]. Naturellement, ccs hypothèses restent, à ce stade, une construction de

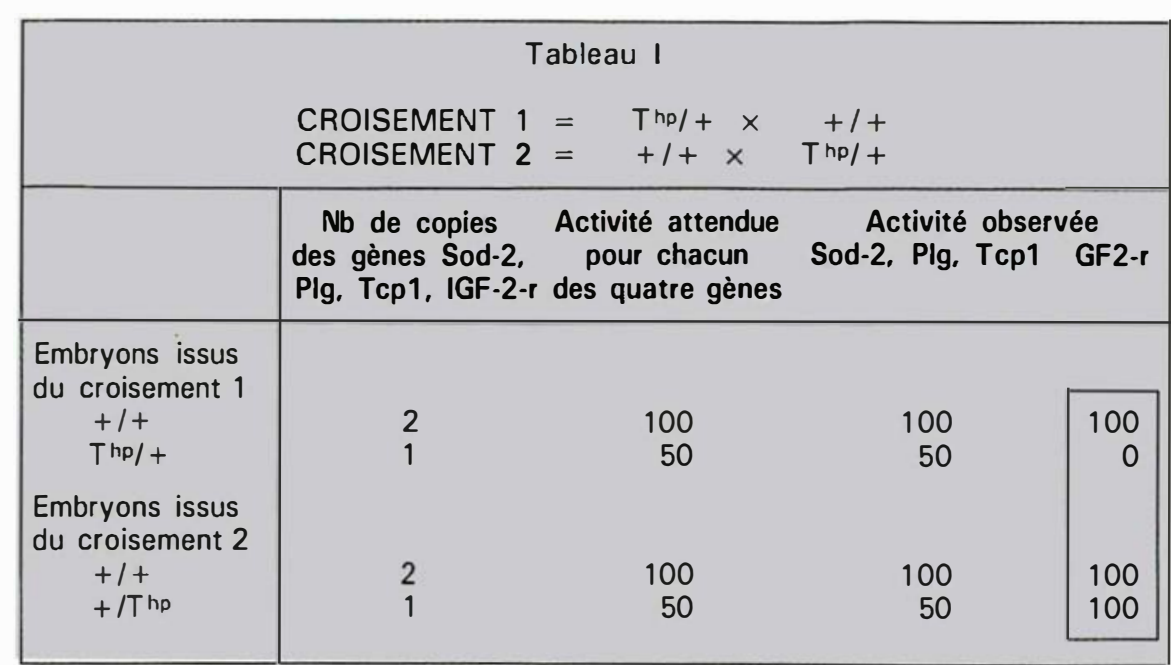

Sod-2: superoxyde dismutase 2; Plg: plasminogène; Tcp 1: T complex protein 1 ; IGF-2-r : récepteur d'IGF-2. 
l'esprit qu'il nous a semblé néanmoins intéressant de proposer aux lecteurs de $\mathrm{m} / \mathrm{s}$ tant est surprenante l'association d'une empreinte parentale de deux gènes codant pour un récepteur et son ligand, et si étrangement cohérente est cette association avec la théorie de Moore et Haig.

C.B.

A.K.

1. Babinet C, Barra J, Renard JP. L'indispensable complémentarité des génomes parentaux. médecine/sciences $1989 ; 5: 8-15$.

2. Paldi A, Jami J. L'empreinte génomique : complémentarité fonctionnelle des deux génomes parentaux. médecine/sciences 1991; 7 : 247-54.

3. Surani A, Reik W, Allen ND. Transgenes as molecular probes for genomic imprinting. Trends Genet 1988 ; 4 : 59-62.

4. Barlow DP, Stöger R, Herrmann BG, Sailo K, Schweifer N. The mouse insulin-like growth factor type 2 receptor is imprinted and closely linked to the tme locus. Nalure 1991 ; 349: 84-87.

5. De Chiara TM, Efstratiadis A, Robertson EL. A growth-deficiency phenotype in heterozygous mice carrying an insulin-like growth factor II gene disrupted by gene targeting. Nalure 1990 ; $345: 78-80$.

6. Moore T, Haig D. Genomic imprinting in mammalian development : a parental tug-ofwar. Trends Genet 1991; 7 : 45-9.

Note ajoutée aux épreuves

L'existence d'une empreinte parentale du gène $I G F-2$ est maintenant bien établie. [De Chiara TM et al. Cell $1991 ; 64$ : 849-60].

\section{Une lésion moléculaire sur le chromosome 21 dans la maladie d'Alzheimer}

L'annonce de la découverte d'une lésion moléculaire dans la maladie d'Alzheimer par l'équipe dirigée par T. Hardy et R. Williamson [1] a fait sensation, car c'est la première fois qu'une anomalie précise est décrite dans cette affection. Il importe de replacer cette avancée dans le cadre général de la maladie, afin de déterminer dans quelle mesure doivent changer les concepts destinés à orienter désormais la recherche dans un domaine dont $\mathrm{m} / \mathrm{s}$ a parlé à de nombreuses reprises*.

On sait que dans le cerveau des sujets atteints de maladie d'Alzheimer s'accumule un peptide de 42 acides aminés au niveau des plaques et des pelotons neurofibrillaires. Ce peptide dit A4 ou $\beta$-amyloïde dérive d'une protéine appelée précurseur de la protéine amyloïde ou APP ; celui-ci se présente sous plusieurs formes, provenant d'épissages différentiels du même gène, dont les principales sont appelées suivant le nombre d'acides aminés de leur séquence, APP 695, 751 ou 770 ; les deux dernières possédant une séquence dotée des propriétés d'un inhibiteur de protéases $\left(\mathrm{m} / \mathrm{s} n^{\circ} 5\right.$, vol. 4, p. 323). Le gène de l'APP est situé sur le chromosome 21.

L'APP a été directement mise en cause dans une autre maladie avec dépôts amyloïdes, l'hémorragie cérébrale avec amyloïdose $\left(\mathrm{m} / \mathrm{s} n^{\circ} 7\right.$, vol. 6, p. 704). Cette observation a contribué à inciter Goate et al. [1], une équipe anglo-américaine constituée de 19 signataires, à analyser

\footnotetext{
* Articles ou nouvelles parues antérieurement dans $\mathrm{m} / \mathrm{s}$ concernant la maladie d'Alzheimer: $n^{\circ} 5$, vol. 3, p. 254 et $256 ; n^{\circ} 10$, vol. 3, p. $620 ; n^{\circ} 5$, vol. 4, p. 323 : n० 9, vol. 5, p. 689 el 690 ; $n^{\circ} 3$, vol. 6, p. $312 ; n^{\circ} 6$, vol. 6, p. $602 ; n^{\circ} 9$, vol. 6, p. 923 .
}

l'ADNc de l'APP 770 en employant avant tout la méthode d'amplification de l'ADN dite PCR. Ils se sont adressés à des formes familiales et ont concentré leurs efforts sur une famille anglaise dont deux frères avaient épousé deux sœurs. Chez les malades, et eux seuls, ils ont trouvé une mutation ponctuelle unique, au nucléotide 2149 de l'ADNc, entraînant un remplacement d'une valine par une isoleucine. Cette mutation se plaçait deux acides aminés en aval par rapport à l'extrémité C-terminale du peptide amyloïde, en position 717 , donc à l'intérieur de la membrane (figure 1). Bien que la modification Val-Ile soit dite conservatrice (elle ne change pas la charge et peu la conformation), le résultat prévisible de cette substitution est d'augmenter l'hydrophobicité du domaine transmembranaire et de renforcer l'ancrage de l'APP à la membrane. Ainsi se comprendrait la rupture de la molécule protéique au niveau de l'extrémité C-terminale du peptide amyloïde, ce qui ne semble pas être le processus normal de dégradation de l'APP $\left(\mathrm{m} / \mathrm{s} n^{\circ} 6\right.$, vol. 6, p. 602). La même mutation a été trouvée dans une autre famille, américaine, et non apparentée à la première. Comme elle ne l'a pas été dans 17 autres familles examinées, dont l'anomalie était pourtant liée au chromosome 21, une hétérogénéité génétique de la maladie d'Alzheimer apparaît évidente, même dans les formes familiales. Cette hétérogénéité était déjà connue, car elle était le seul élément indiscutable qui ressortait d'une vaste enquête portant sur 48 familles et dont $\mathrm{m} / \mathrm{s}$ a rendu compte en novembre 1990 ( $n^{\circ} 9$, vol. 6, p. 923). L'hétérogénéité peut se situer à deux niveaux. 